\title{
Optimierungsverfahren für schaltungstechnische Realisierungen zellularer Rechner
}

\author{
G. Geis, F. Gollas, and R. Tetzlaff \\ Institut für Angewandte Physik, Johann Wolfgang Goethe-Universität, Frankfurt, Deutschland
}

Zusammenfassung. Bei intelligenten Sensoren soll die Aufnahme von Signalen und deren, zumindest teilweise durchgeführte, Verarbeitung mit einer einzigen Anordnung erfolgen. Dazu steht häufig eine elektronische Schaltung zur Verfügung, die allerdings zur Einhaltung von Echtzeitbedingungen nur für eine relativ einfache Signalverarbeitung verwendet werden kann. Einen möglichen Ausweg bildet die Verwendung parallel arbeitender Rechnersysteme. In dieser Hinsicht sind programmierbare Schaltungen mit z.B. optischen Sensor-Anordnungen besonders interessant, die auf Zellularen Nichtlinearen Netzwerken basieren. Derartige miniaturisierte Systeme eröffnen aufgrund ihrer zellularen Architektur neue Möglichkeiten zur Signalverarbeitung mit einem Leistungsvermögen, das im Bereich von TeraOperationen pro Sekunde liegt. Für viele aktuelle Problemstellungen wäre es von Vorteil, wenn diese zellularen Systeme eigenständig Parameteradaptionen durchführen könnten. Eingangssignale, die beispielsweise über die vorhandenen optischen Sensoren aufgenommen werden, führten dann zu einer Neuberechnung bzw. Anpassung der Netzwerksparameter. Aufgrund der beachtlichen Leistungsfähigkeit solcher Schaltungen wäre damit die Möglichkeit gegeben, eine adaptive Signalverarbeitung bei zeitlich veränderlichen Problemen vorzunehmen. In diesem Beitrag wird die Implementierung und Analyse von Lernverfahren auf dem EyeRISTM System, das einen zellularen Prozessor ACE16kv2 ${ }^{\mathrm{TM}}$ mit $128 \times 128$ Zellen enthält, zur adaptiven Parameterbestimmung betrachtet. Anhand verschiedener Problemstellungen aus dem Bereich der Bildverarbeitung werden unterschiedliche Lernverfahren verglichen und deren Leistungsfähigkeit untersucht.

Schlagworte: Intelligente Sensoren, Zellulare Nichtlineare Netzwerke, Optimierungsverfahren, EyeRis visual system

Correspondence to: G. Geis

(geis@iap.uni-frankfurt.de)

\section{Einleitung}

Im Jahre 1988 führten (Chua und Yang, 1998) die Zellularen Neuronalen/Nichtlinearen Netzwerke (englisch Cellular Neural/Nonlinear Networks, abgekürzt CNN) ein. Die zugrunde liegende Idee besteht darin, Zellen zu einem nur lokal gekoppelten Netzwerk mit einer nicht linearen Ausgangsfunktion zusammenzufügen, die der Zustandsgleichung

$\frac{d x_{i j}}{d t}=-x_{i j}+\sum_{k, l \in \mathcal{N}_{i j}} \mathbf{A} x_{k l}+\sum_{k, l \in \mathcal{N}_{i j}} \mathbf{B} y_{k l}+z$

folgen und als Nichtlinearität die stückweise lineare Kennlinie

$y_{i j}=\frac{1}{2}\left(\left|x_{i j}+1\right|-\left|x_{i j}-1\right|\right)$

realisieren. Jede einzelne Zelle des Netzwerks ist, wie Abb. 1 illustriert, nur lokal in einer meist kleinen Nachbarschaft $\mathcal{N}$, welche gegeben ist als die Menge aller Zellen, die innerhalb eines durch den Radius $r$ definierten Einflussgebietes liegen,

$$
\begin{aligned}
\mathcal{N}_{i j}= & \left\{C_{k l}: \max (|k-i|,|l-j|) \leq r,\right. \\
& 1 \leq k \leq M, 1 \leq l \leq N\}
\end{aligned}
$$

gekoppelt.

Die in Abb. 1 gezeigten gewichteten Zellverknüpfungen werden häufig bei translationsinvarianten Netzwerken in Matrixschreibweise

$\mathbf{A}=\left(\begin{array}{ccc}a_{-1,-1} & a_{-1,0} & a_{-1,1} \\ a_{0,-1} & a_{0,0} & a_{0,1} \\ a_{1,-1} & a_{1,0} & a_{1,1}\end{array}\right), \quad \mathbf{B}=\left(\begin{array}{cccc}b_{-1,-1} & b_{-1,0} & b_{-1,1} \\ b_{0,-1} & b_{0,0} & b_{0,1} \\ b_{1,-1} & b_{1,0} & b_{1,1}\end{array}\right)$

dargestellt und dann als Templates bezeichnet. Die Matrix A wird hierbei auch als Feedback-Template bezeichnet; sie dient zur Festlegung der Kopplungsstärke zwischen den Ausgängen einzelner Zellen. Die Matrix B legt als sogenanntes Feedforward-Template die Gewichte der Eingänge

Published by Copernicus Publications on behalf of the URSI Landesausschuss in der Bundesrepublik Deutschland e.V. 


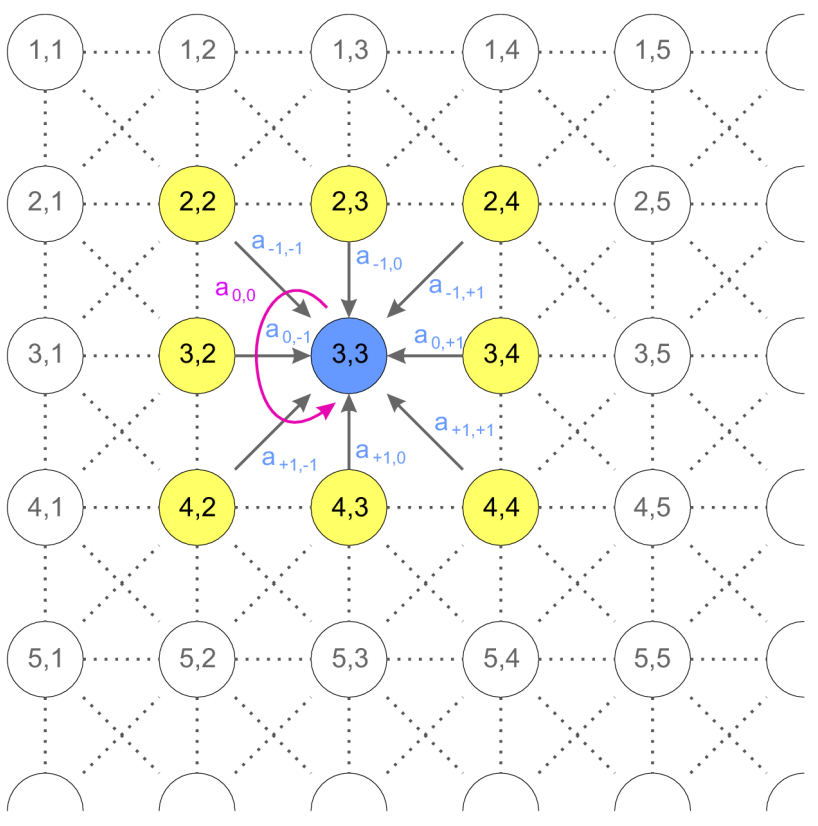

Abb. 1. Einfluss der Feedback-Template auf die Zelle $C_{3,3}$ in einem zweidimensionalen CNN mit direkter Nachbarschaft $(r=1)$.

fest. Die Größe $z$ in Gl. 1 repräsentiert einen Grundbeitrag auch Bias genannt, der im allgemeinen für alle Zellen des Netzwerks gleich ist. Durch die lokale Kopplung von Zellen eignen sich solche Netzwerke hervorragend für eine schaltungstechnische Realisierung. Das kürzlich vorgestellte EyeRISTM System enthält mit dem ACE16kv2 ${ }^{\mathrm{TM}}$ einen sogenannten Focal Plane Prozessor(FPP) mit $128 \times 128$ Zellen und optischen Sensoren, welcher von der Firma ANAFOCUS (Anafocus Ltd. Avd. Isaac Newton s/n) entwickelt wurde. Das EyeRISTM System kombiniert hierbei die Programmierbarkeit einer herkömmlichen digitalen CPU mit einer analogen hochgradig parallelen Verarbeitung anhand von zellularen Netzwerken (Roska, 2000). Der digitale Prozessor wird durch ein FPGA realisiert, welches zu diesem Zweck als eine CPU mit dem NIOS II Kern der Firma Altera konfiguriert wurde. Dieser Prozessor dient der Kontrolle des analog arbeitenden ACE16kv2 ${ }^{\mathrm{TM}}$, der Nachverarbeitung von Daten und als Bindeglied zur Kommunikation mit einem PC. Der ACE16kv2 ${ }^{\text {TM }}$ bietet durch seine massive Parallelität bei einer Größe von ca. $1 \mathrm{~cm}^{2}$ die Rechenleistung eines Großrechners. Durch seine Funktionsweise, sowohl als Sensoranordnung zur Aufnahme von Bildern als auch der parallelen Weiterverarbeitung zu dienen, bildet der ACE16kv2 ${ }^{\mathrm{TM}}$ einen "intelligenten" Sensor. Dieser kann dank seiner der Programmierbarkeit für zahlreiche Aufgabenstellungen verwendet werden und im Speziellen mittels eines Optimierungsverfahrens zu einem adaptiven System erweitert werden. Algorithmen zur Optimierung (Kunz und Tetzlaff, 2000; Storn und Price, 1995) welche auf dem EyeRISTM System implementiert wurden, werden im folgenden vorgestellt, außerdem deren

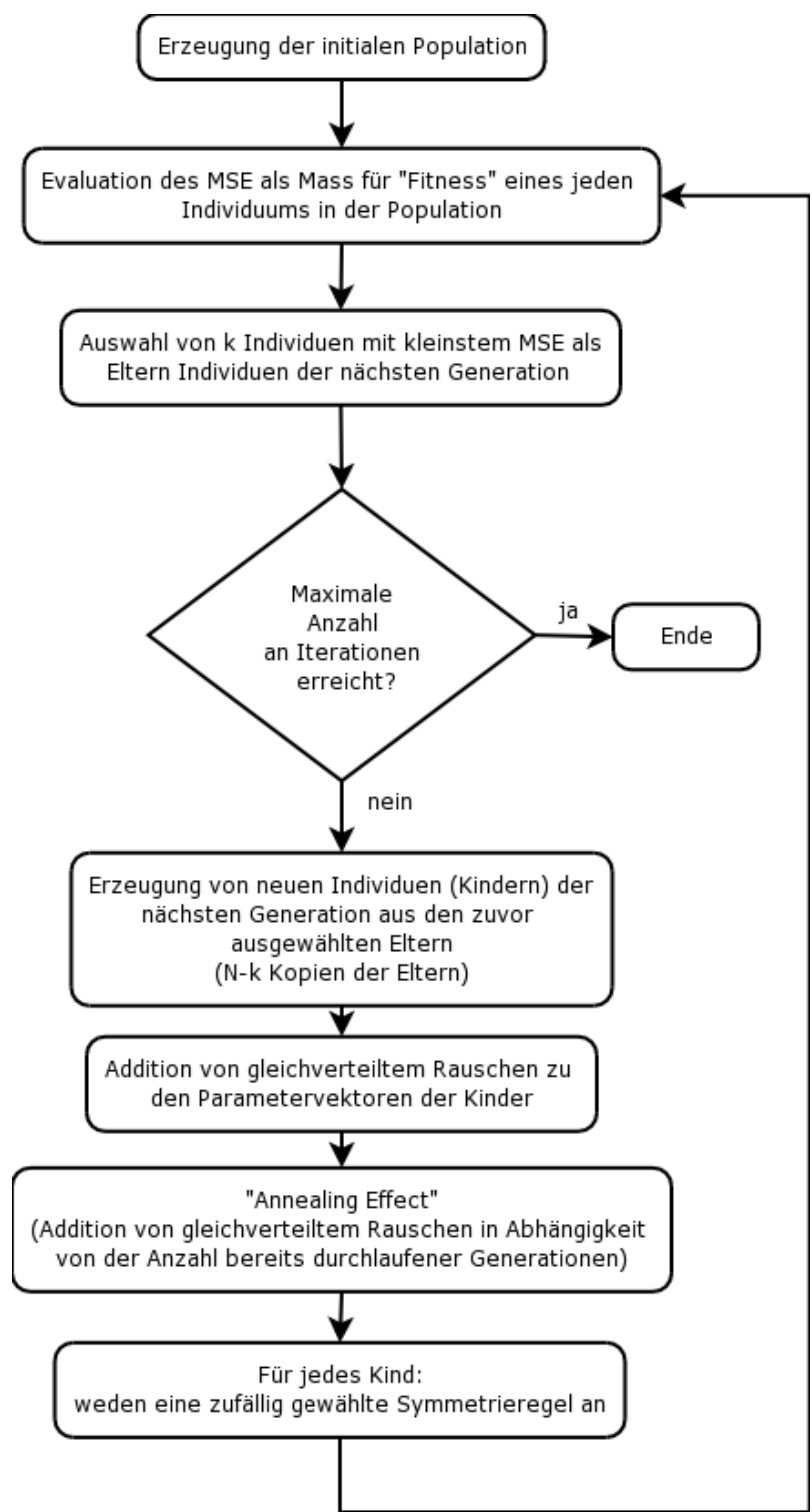

Abb. 2. Flußgraph des SSAPE Algorithmus. In jedem Iterationsschritt werden $k$ Individuen mit kleinstem MSE der Gesamtmenge aller Individuen einer Generation ausgewählt. Diese bilden die Eltern der nächsten Generation. Neue Parametervektoren (Kinder) werden als Kopien der Eltern angelegt und durch Rauschen und anwenden von zufällig gewählten symmetrieerzeugenden Regeln mutiert.

Resultate gezeigt und diskutiert.

\section{Evolutionäres Lernverfahren}

Ein Algorithmus zur Optimierung von Parametern für schaltungstechnische Realisierungen von CNN, sollte auch zusätzliche, die schaltungstechnische Realisierung betreffende Parameter berücksichtigen. Im Falle des Eye- 

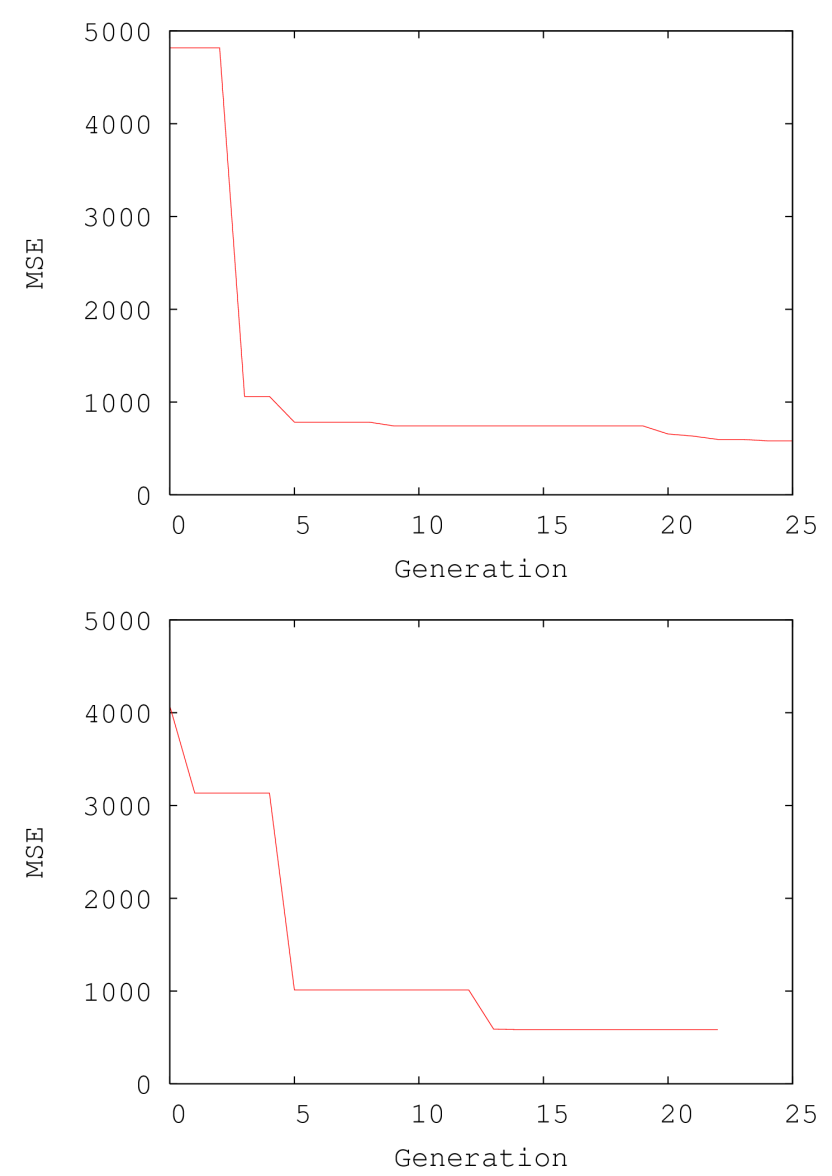

Abb. 3. Zeitliche Entwicklung des MSE als Funktion der Generationen (Iterationsschritte) für DE (oben) und SSAPE (unten) Algorithmus. (11 Parameter).

RIS $^{\text {TM }}$ Systems betrifft das Einstellungen von Spannungsbereichen, welche den Zustand, die Eingaben, sowie die Kopplungsgewichte repräsentieren. Der analoge Prozessor ACE16kv2 ${ }^{\mathrm{TM}}$ enthält für eine Verbindung zu einem digitalen System bereits D/A- und A/D-Wandler mit einer Auflösung von 8bit. Die in diesem Beitrag vorgestellten Verfahren verwenden diesen mit 8bit darstellbaren Zahlenbereich zur Darstellung von Parametern, weshalb keine weitere Umrechnung, z.B. von einer Fließkommadarstellung für die Gewichtswerte und umgekehrt, nötig ist. Allein diese Maßnahme beseitigt bereits eine mögliche Quelle von Ungenauigkeiten, durch Vermeidung von Rundungsfehlern, die bei der Umrechnung auftauchen könnten. Für die Untersuchungen wurde der mittlere quadratische Fehler (MSE)

$M S E=\frac{1}{M} \sum_{i=1}^{128} \sum_{j=1}^{128}\left(x_{i, j}-\hat{x}_{i, j}\right)^{2}$

als Fehlermaß herangezogen, wobei $M=(128 \cdot 128)$ die Gesamtzahl der Zellen im Netzwerk, $x_{i, j}$ den jeweiligen Zellausgangswert und $\hat{x}_{i, j}$ den Referenzwert an der Position $i, j$
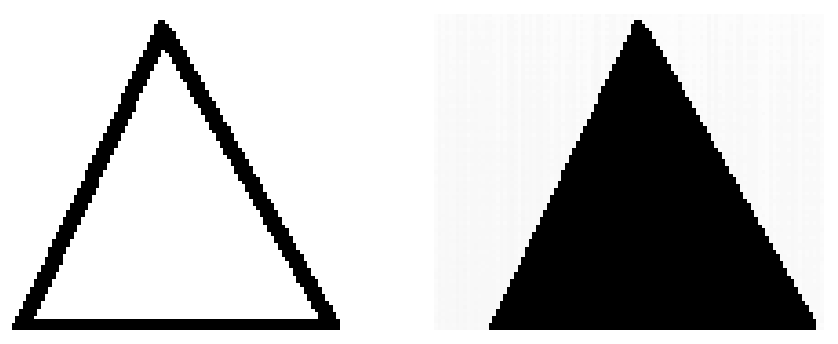

Abb. 4. Beispiel für die Hole-Filler Verarbeitung; links: Anfangszustand; rechts: Resultat nach Anwendung der Optimierung auf dem EyeRIS ${ }^{\text {TM }}$ System.

bezeichnet, weshalb der maximale $M S E$ für die vorgegebene Auflösungsgenauigkeit von 8 bit durch

$M S E_{\max }=\frac{1}{128^{2}} \sum_{i=1}^{128} \sum_{j=1}^{128} 255_{i, j}^{2}=255^{2}$.

gegeben ist.

Der hier vorgestellte Symmetry-Supported-AnnealedParameter-Evolution (SSAPE) Algorithmus wurde in Hinblick auf eine gewünschte Robustheit gegenüber Bauteiletoleranzen unter Berücksichtigung der räumlichen $\mathrm{CNN}$ Struktur speziell für das EyeRISTM System entwickelt. Ein Parametervektor kann hierbei zum einen aus den Gewichtswerten der Zellen und zum anderen aus Parametern, welche die Funktionsweise der elektrischen Schaltungen der Zellen bestimmen, zusammengesetzt sein. Da der SSAPE Algorithmus evolutionär inspiriert ist, wird ein Parametervektor, im folgenden auch als Individuum bezeichnet. Werden mehrere Individuen zu einer Menge zusammengefaßt, so spricht man auch von einer Population. Betrachtet man diese Population zu einem Zeitpunkt $t$, so wird diese Menge auch Generation zum Zeitpunkt $t$ genannt. In jedem Iterationsschritt wird eine Untermenge von $k$ Individuen (Eltern) der Gesamtmenge aller Individuen einer Generation der Mächtigkeit $N$ in Abhängigkeit des MSE des jeweiligen Parametersatzes ausgewählt. Die Individuen der folgenden Generation $t+1$ werden aus den zuvor ausgesuchten $k$ Individuen der Generation zum Zeitpunkt $t$ gebildet, indem diese vervielfältigt und mit weißem Rauschen überlagert werden, bis wieder $N$ Individuen gebildet wurden. Ein von der aktuellen Iteration abhängiges Rauschen wirkt zusätzlich auf die Individuen ein, wobei dieser Einfluß bei jeder folgenden Iteration geringer wird, was einem „Auskühleffekt”, bei einem Annealing, entspricht. Die neuen Individuen (Kinder) werden zusätzlich mit weiteren zufällig gewählten Symmetrie erzeugenden Regeln modifiziert. Diese Regeln basieren darauf, daß den meisten Anwendungen von CNN eine Symmetrie bezüglich der „Template”- Matrizen zugrunde liegt. Die Symmetrie erzeugenden Operationen sind für eine $3 \times 3$ Matrix unter anderem Spiegelungen an der mittleren Spalte oder mittleren Zeile, bzw. eine punktsymmetrische Spiegelung am mittleren Element. Durch die Selektion der $k$ Individuen einer Generati- 

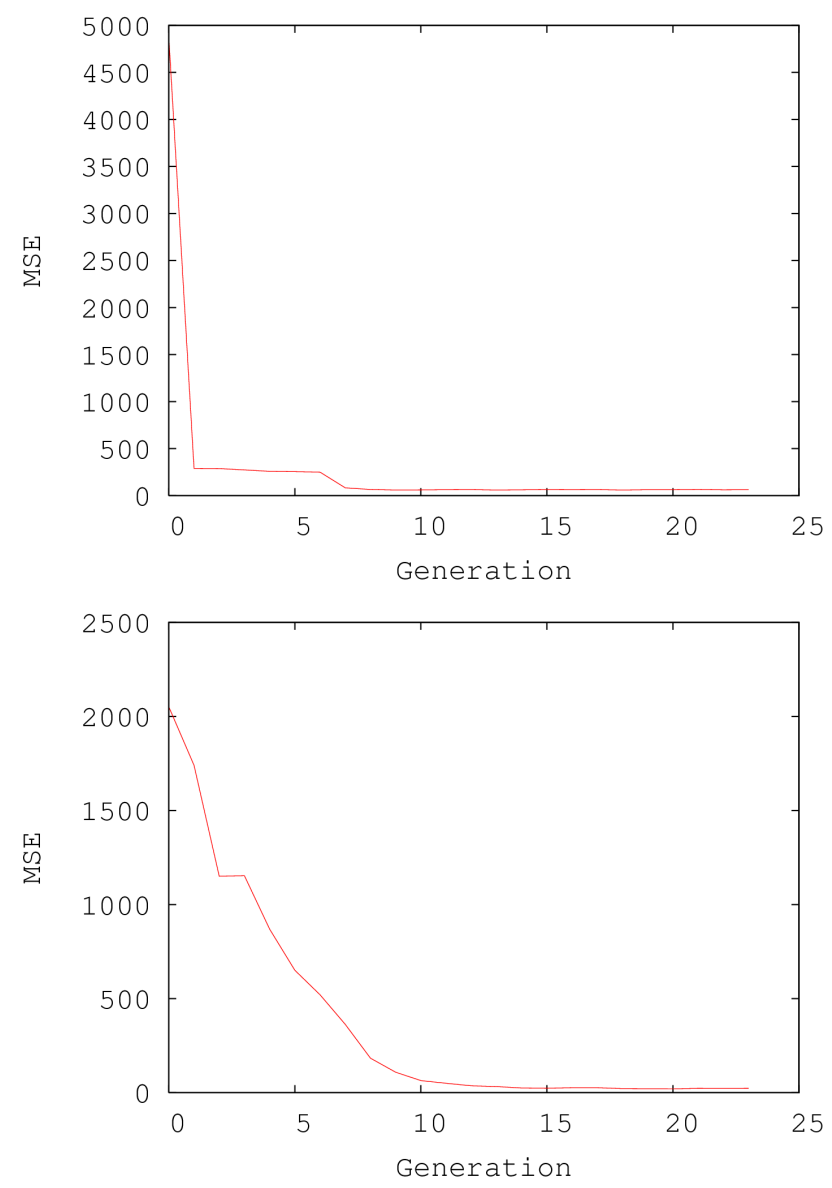

Abb. 5. Kantendetektion: 9 Parameter (oben); 13 Parameter (unten).

on mit den jeweils kleinsten Fehlern bildet sich eine Population aus, in welcher eine Symmetrie bevorzugt wird. Diese Symmetrie ist nicht fest vorgegeben, sondern wird durch das Optimierungsverfahren erzeugt, was insbesondere dann von Vorteil ist, wenn Bauteiletoleranzen eine feste Vorgabe von Symmetrien behindern.

Die Leistungsfähigkeit des SSAPE Algorithmus wird in diesem Beitrag mit einem anderen evolutionär inspirierten Verfahren, dem Differential Evolution (DE) Verfahren, erstmals von Storn und Price $(1995,1996)$ eingeführt, verglichen. Dazu wurde der DE Algorithmus auf dem EyeRIS $^{\text {TM }}$ System implementiert. Dieser Algorithmus bildet jedes Individuum der folgenden Generation aus mehreren zufällig gewählten Individuen der momentanen Generation. In einer seiner einfachen Implementierungen wird für die Erzeugung eines neuen Parametervektors der folgenden Generation zuerst aus zwei Parametervektoren der momentanen Generation eine gewichtete Differenz gebildet. Diese wird anschließend zu einem weiteren Parametervektor der momentanen Generation unter Berücksichtigung eines Crossover-Operators (Storn und Price) addiert.

\section{Ergebnisse}

In diesem Abschnitt werden Ergebnisse gezeigt, die aus der Anwendung der vorgestellten Optimierungsverfahren auf einfache Problemstellungen der Bildverarbeitung mit CNN resultieren. Dies betrifft, die sogenannte „Hole-Filler Tem-

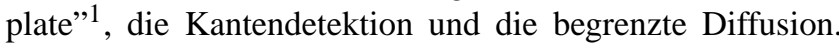
Die Hole-Filler Operation stellt eine propagierende Operation dar, d.h., daß die Rechenzeiten abhängig von der Fläche des zu füllenden Objekts verschieden sein können.

Bei der binären Kantendetektion werden sämtliche Bildpixel, die nicht die Kante eines Bildobjekts darstellen, zu ,,weißen” Pixeln im stabilen Endzustand des Netzwerks. Die begrenzte Diffusion ist eine schwierigere Problemstellung. Hier wird als Eingabe ein Grauwertbild und als Anfangszustand ein binärwertiges Bild, welches kleine schwarze Markierer (einige wenige schwarze aneinandergrenzende Pixel) enthält, verwendet. Findet sich im Anfangszustand ein Markierer an einer Stelle, welche im Eingabebild korrespondierend ein Bildobjekt enthält, so breitet sich dieser Markierer auf angrenzende Pixel aus. Der Grauwert des Pixels des Bildes im Eingang einer jeden Zelle beeinflußt hierbei den Grad der Ausbreitung. Ist der Grauwert im Eingang niedriger als ein durch den Bias $z$ zu beeinflussender Wert, dann werden Markierer an solchen Stellen im Verlauf der analogen Operation ausgelöscht.

Während der Optimierung wird jeder Parametersatz einer Generation auf dem EyeRIS ${ }^{\mathrm{TM}}$ System unter Verwendung unterschiedlicher Bilder, welche die sogenannte Trainingsmenge bilden, zur Berechnung herangezogen; dabei wird der jeweilige MSE ermittelt. Anschließend werden zur Überprüfung die optimierten Parameter auf Bilder angewandt, welche nicht in der Trainingsmenge enthalten sind. In Abb. 3 ist die zeitliche Entwicklung des MSE gegen den Iterationsschritt (Generation) für die Hole-Filler Verarbeitung gezeigt.

Wie erkennbar ist, nimmt der MSE bei Anwendung des DE Algorithmus anfangs stark ab und verweilt dann lange auf einem Fehlerniveau. Erst nach der 18ten Generation ist eine weitere Absenkung des MSE zu beobachten. Ein ähnliches Verhalten ist auch beim SSAPE Algorithmus zu beobachten. In Abb. 4 ist ein Ergebnis gezeigt, welches nach Anwendung eines optimierten Parametersatzes auf ein nicht in der Trainingsmenge enthaltenes Bild resultiert.

Abbildung 5 bietet einen Vergleich zur Kantendetektion, für die Konvergenz des SSAPE Algorithmus mit unterschiedlicher Anzahl zu optimierender Parameter.

In den bisher betrachteten Fällen zeigte sich der Trend mehr Iterationsschritte zu benötigen, um einen vergleichbar guten Wert des MSE zu erreichen, wenn mehr Parameter zu optimieren waren. Im gezeigten Fall von 9 Parametern kann bereits ein Abfall des MSE nach der 5. Generation beobachtet werden, während im dargestellten Fall von 13 zu optimie-

\footnotetext{
${ }^{1}$ Dabei werden Objekte eines binären Bildes mit ,,schwarzen Pixeln" ausgefüllt.
} 


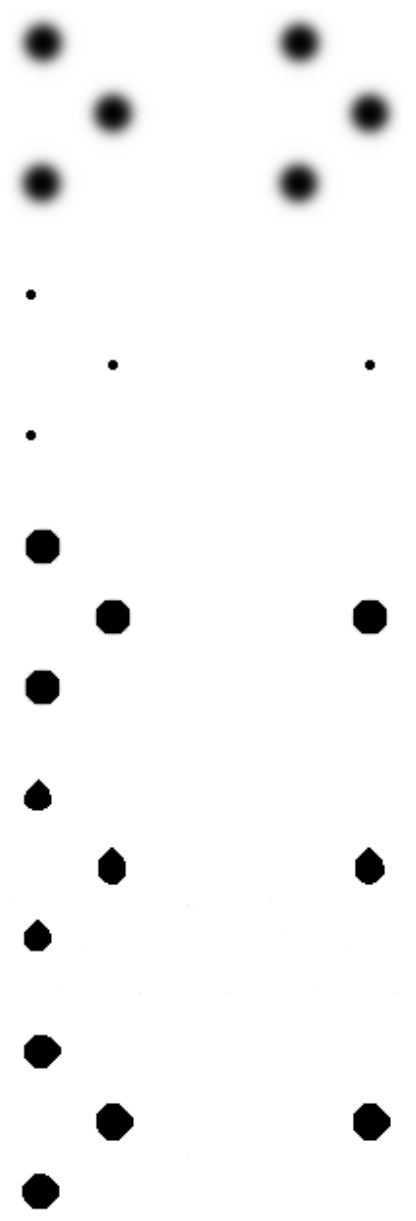

Abb. 6. Von oben nach unten: Bild in der Eingabe des CNN, Anfangszustand des CNN, Referenzbild, Ergebnis des besten Individuums in der 3.Generation, folgend: Ergebnis des beste Idividuums in der 23.Generation.

renden Parametern bereits 10. Generationen nötig sind um einen deutlichen Abfall des MSE zu erreichen.

Die Ergebnisse der Optimierung der begrenzten Diffusion sind in Abb. 6 in zwei Spalten gezeigt. In beiden Fällen wurde das gleiche Eingangsbild benutzt, während für den Anfangszustand zwei unterschiedliche binäre Bilder verwendet wurden. Während in der rechten Spalte der Anfangszustand aus nur einem Markierer besteht, wurden für den in der linken Spalte gezeigten Fall drei Markierer verwendet. Zum Vergleich sind Ergebnisse eines Trainings für die 3. Generation, welche schon zu einem guten Resultat führt, und der 23. Generation gezeigt, welche nahe an das gewünschte Resultat heranreicht. Die zeitliche Entwicklung des MSE des jeweils besten Parametersatzes einer Generation ist in Abb. 7 gezeigt.

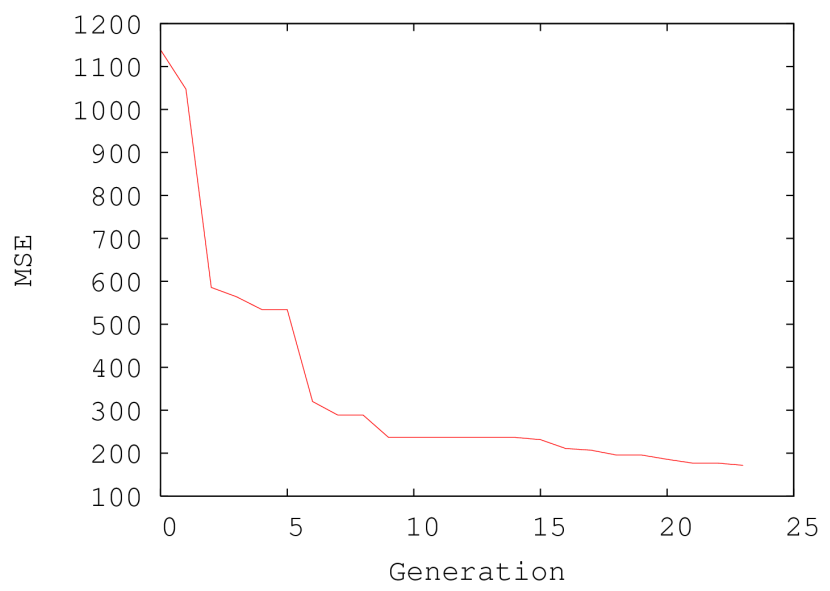

Abb. 7. MSE in Abhängigkeit von den Generationen für die Optimierung zur begrenzten Diffusion.

\section{Diskussion}

In diesem Beitrag wurden zwei Optimierungsverfahren für das EyeRISTM System mit seinem analogen ACE16kv2 ${ }^{\text {TM }}$ betrachtet, welche die gegebenen Eigenschaften des Systems mitberücksichtigen. Das SSAPE Verfahren, welches die räumlichen Symmetrieeigenschaften berücksichtigt, wurde vorgestellt und diskutiert. Ein zweites Verfahren, das Differential Evolution (DE), wurde zum Vergleich, ebenfalls auf das EyeRISTM System übertragen und auf Problemstellungen der Bildverarbeitung angewandt. In einem weiteren Anwendungsfall wurde das SSAPE Verfahren auf die Problemstellung der begrenzten Diffusion angewandt. Hierbei konvergierte das Verfahren schon nach wenigen Iterationsschritten.

Danksagung. Die Autoren bedanken sich bei Prof. Dr. RodríguezVázquez (Anafocus Ldt. Avd. Isaac Newton s/n) und seiner Arbeitsgruppe für die Unterstützung und Zurverfügungstellung des EyeRIS V1.1 $1^{\text {TM }}$ Systems.

\section{Literatur}

Anafocus Ltd. Avd. Isaac Newton s/n, Pabellón de Italia, $7^{a}$ Planta, Ático Parque Tecnológico Isla de la Cartuja 41092 Sevilla, Spain, www.anafocus.com, VISION SYSTEMS ON-CHIP - technology.

Chua, L. O. and Yang, L.: Cellular neural network: Theory an application, IEEE Transactions on Circuit and System, 35, $1257-$ 1290, 1988.

Kunz, R. and Tetzlaff, R.: Evolutionary learning strategies for cellular neural networks. In Proceedings of IEEE Int. Workshop on Cellular Neural Networks and Their Applications, CNNA 2000, volume ISBN 0-7803-6344-2, pages 241-246, 2000.

Roska, T.: Computational and computer complexity of analogic cellular wave computers. in Proceedings of IEEE Int. Workshop on Cellular Neural Networks and Their Applications, CNNA 2000, ISBN 981-238-121-X, 323-335, 2000. 
Storn, R. and Price, K., Differential Evolution (DE) for Continuous Function Optimization, www.icsi.berkeley.edu/ $\sim$ storn/ code.html.

Storn, R. and Price, K.: Differential evolution - a simple and efficient adaptive scheme for global optimization over continuous spaces, Technical report tr-95-012, ICSI, March, 1995.
Storn, R. and Price, K.: Minimizing the real functions of the icec'96 contest by differential evolution, IEEE Conference on Evolutionary Computation, Nagoya, 842-844, 1996. 